\title{
MULTI-BEAM ACCELERATION IN FFAG SYNCHROTRON
}

\author{
Y. Mori, A. Takagi, KEK, Tsukuba, Japan \\ K. Koba, FNAL, Batavia, IL 60510, USA
}

\section{Abstract}

The first acceleration of protons in a FFAG (Fixed Field Alternating Gradient) synchrotron was demonstrated at KEK in June 2000.

Since the magnetic field in the FFAG is static, therefore the repetition rate depends only on the acceleration frequency. We have developed a high gradient rf cavity using the 'FINEMET' magnetic alloy cores. With this new type of rf cavity, the cycle time of the synchrotron can become very high, $1 \mathrm{KHz}$ or more, which is more than 100 times of the ordinary synchrotron. Thus, the FFAG is an attractive machine for accelerating intense beams.

Moreover, the FFAG can accelerate not only single bunch, but also several bunches each having a different energy, by changing the rf frequency for each bunch. The new type rf cavity using the FINEMET cores has a low Q-value which easily enables the coexistence of different frequencies. We have demonstrated this multi-energy, multi-bunch acceleration at the KEK POP FFAG machine.

\section{INTRODUCTION}

The POP (proof-of-principle) FFAG has been developed at KEK, and we have already obtained the circulating beam in this ring[1].

The typical parameters of this machine are listed in Table 1.

The POP FFAG is a radial sector type. The ring consists of eight sectors and each sector has a DFD triplet focusing structure[2].

Table 1: Main parameter of POP FFAG

\begin{tabular}{|l|l|}
\hline Accelerated particle & Proton \\
\hline Injection energy & $50 \mathrm{keV}$ \\
\hline Extraction energy & $500 \mathrm{keV}$ \\
\hline Radial crossed orbit & $0.81-1.14 \mathrm{~m}$ \\
\hline Field index & 2.5 \\
\hline Harmonic number & 1 \\
\hline Repetition rate & $1 \mathrm{kHz}$ \\
\hline RF frequency & $0.61-1.38 \mathrm{MHz}$ \\
\hline RF voltage & $1.3-3.0 \mathrm{kV}$ \\
\hline
\end{tabular}

When the synchronous phase is set to be 20 degree, the requested rf voltage becomes at least $1.5 \mathrm{kV}$ during the $1 \mathrm{msec}$.

We have developed the rf cavity, shown in Fig. 1, using two rectangular 'FINEMET' cores of $1.1 \mathrm{~m}$ (width) $\mathrm{x} 0.7 \mathrm{~m}$ (height). The thickness of the core is $30 \mathrm{~mm}$. The window's opening is $640 \mathrm{~mm}$. The shunt impedance of this core is about $82 \mathrm{ohms}$. The $55 \mathrm{~kW}$ rf amplifier which consists of two tetrodes (Eimac 4CW25,000) is used.

\subsection{Frequency characteristic of the rf cavity.}

The available frequency range of the whole RF system is determined by the characteristic of the impedance matching section to connect the $55 \mathrm{~kW}$ amplifier with $1 \mathrm{~kW}$ driver amplifier, and by the choke inductance to block the rf current to the anode power supply.

We made a test circuit with matching transformer. In order to simulate the real condition, the $200 \mathrm{pF}$ capacitor, which number was assumed tetrode's capacitance, was 
mounted together. To obtain the wide frequency range, we made the transformer using the two kinds of core, 'Nanoperm' and Ni-Zn ferrite. Figure 2 shows the frequency characteristic of this circuit. The resonant frequency was about $5.0 \mathrm{MHz}$.

We also measured the choke inductance which supposed to be more than $1 \mathrm{mH}$ and plotted the result in Fig. 3. The series resonance frequency was about 5.0MHz.

From these two results, the upper limit of the frequency range was $5 \mathrm{MHz}$. Since the rf frequency is from $0.6 \mathrm{MHz}$

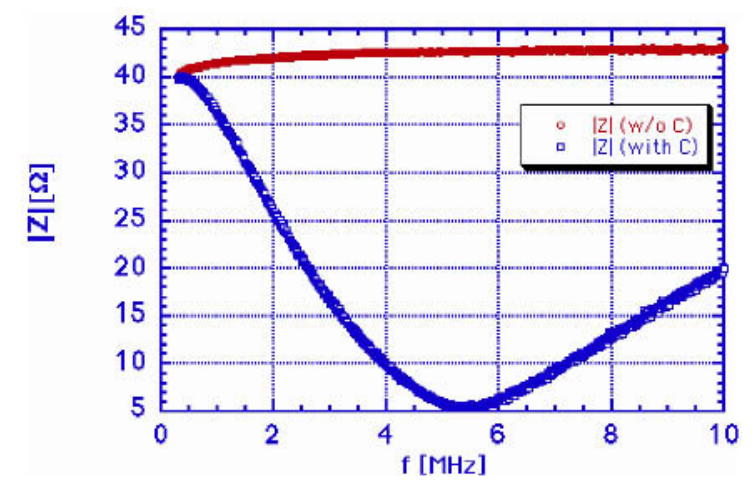

Figure 2: The frequency characteristic of the matching transformer as a function of the frequency.

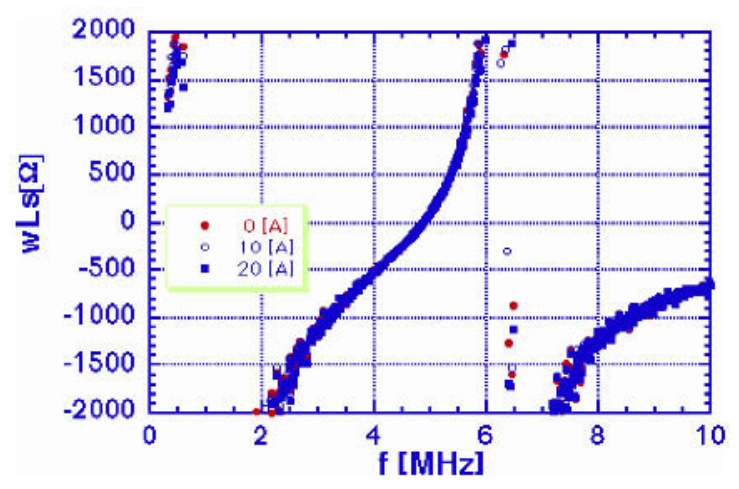

Figure 3: The choke inductance which supposed to be more than $1 \mathrm{mH}$. The series resonance frequency was about $5.0 \mathrm{MHz}$.

to $1.2 \mathrm{MHz}$, it is possible to mix the higher harmonics up to $\mathrm{h}=3$.

\section{SINGLE BUNCH ACCELERATION}

In the first trial of beam acceleration, since the $\mathrm{rf}$ voltage was fixed to $1.66 \mathrm{kV}$ during the acceleration from $50 \mathrm{keV}$ to $374 \mathrm{keV}$, the rf frequency has to be changed from $624 \mathrm{kHz}$ to $1.25 \mathrm{MHz}$ as shown in Fig. 4 .

The circulating beam signals were observed with the beam position monitor which consist of two electrodes.
The observed revolution frequency was changed from $610 \mathrm{kHz}$ to $1.251 \mathrm{MHz}$ with accuracy of $6.1 \mathrm{kHz}$. The measured synchrotron frequency was changed from $24.06 \mathrm{kHz}$ to $16.78 \mathrm{kHz}$ with accuracy of $6.1 \mathrm{kHz}$. These synchrotron frequencies agree well with the calculated values assuming with small amplitude approximation.

\section{MULTI BUNCHES ACCELERATION}

There is a possibility to accelerate several bunches each having a different energy, by applying the different rf

[injection]

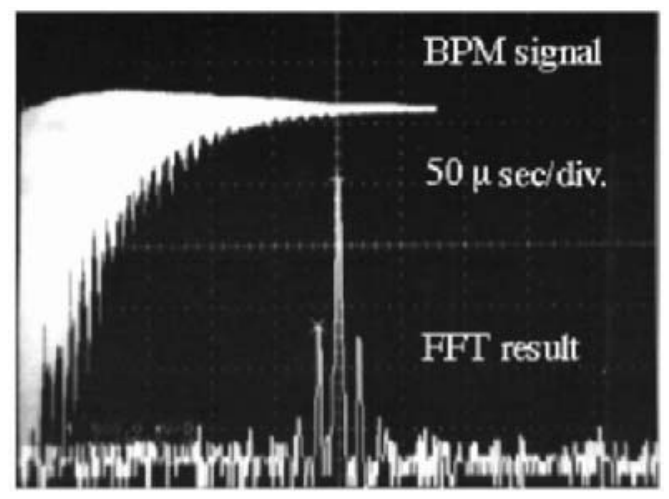

revolution frequency: $\quad 0.610 \mathrm{MHz}$ synchrotron frequency: $\quad 24.06 \mathrm{kHz}$

[flat top]

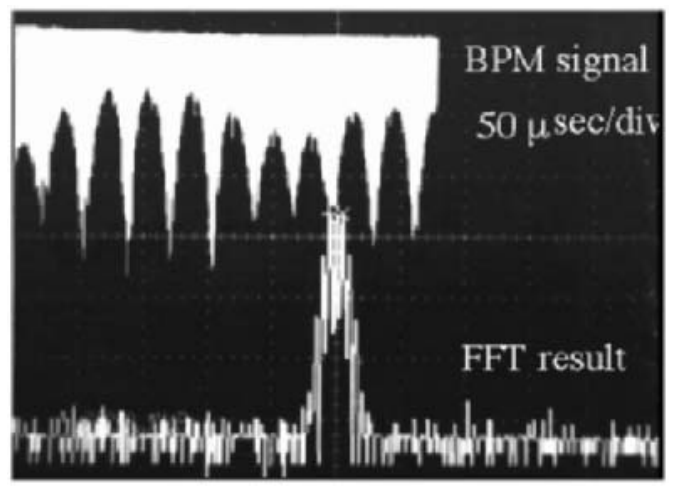

revolution frequency: $\quad 1.251 \mathrm{MHz}$

synchrotron frequency: $16.78 \mathrm{kHz}$

Figure 4: The first trial of beam acceleration, since the rf voltage was fixed to $1.66 \mathrm{kV}$ during the acceleration from $50 \mathrm{keV}$ to $374 \mathrm{keV}$, the rf frequency has to be changed from $624 \mathrm{kHz}$ to $1.25 \mathrm{MHz}$.

frequency for each bunch. The rf cavity has a low Qvalue which easily enables the coexistence of different frequencies. We have demonstrates two bunches acceleration at the KEK POP FFAG machine. The first bunch was accelerated with frequency 1 (f1) and second bunch was accelerated with frequency 2 (f2). Since there were two frequencies simultaneously, the first rf voltage 
applied to first bunch affects to second bunch. If we consider that the bucket separatorix doesn't touch to each other, the frequency separation, $\mathrm{df}=\mathrm{f} 1-\mathrm{f} 2$ is written by equation(1).

$$
\Delta f \geq 4 * f_{s y}
$$

fsy is the synchrotron frequency.

In the POP FFAG, the synchrotron frequency was around $20 \mathrm{kHz}$. We kept the frequency separation was $100 \mathrm{kHz}$ and accelerated two bunches with the rf function shown in Fig. 5.

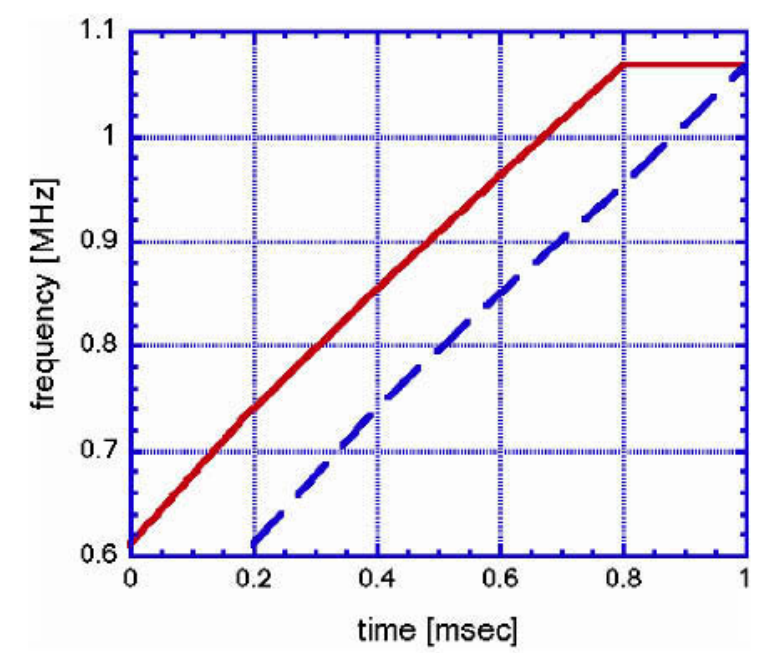

Figure 5: The rf function of the two bunch acceleration. In the POP FFAG, We kept the frequency separation of $100 \mathrm{kHz}$.

After the acceleration, there are two frequency peaks on the beam signal as shown in Fig. 6. These frequencies, $1068.12 \mathrm{kHz}$ and $984.192 \mathrm{kHz}$, consist with the rf frequencies which are applied to two bunches.

\section{CONCLUSION}

We have demonstrated the two bunch acceleration at the KEK POP FFAG accelerator with a newly developed wide band rf cavity using FINEMET magnetic alloy cores. Using this scheme, the beam duty factor in FFAG accelerator increases effectively and an high intensity beam acceleration can be realized .

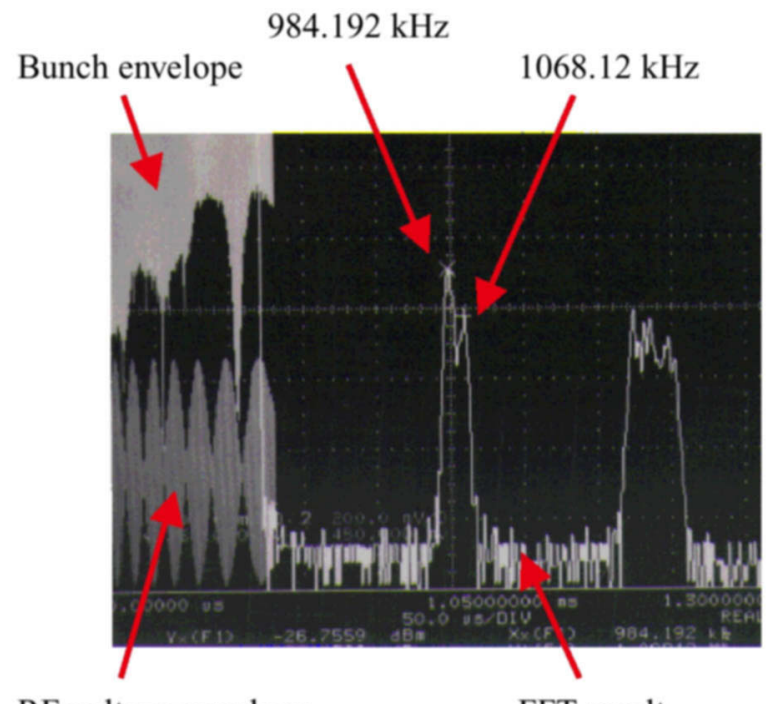

RF voltage envelope

FFT result

Figure 6: Observed beam signal. There are two frequency peaks on the beam signal after two bunch acceleration.

\section{REFERENCES}

[1] M. Aiba et al., Proc. of EPAC2000, Vienna, (2000) 581-583.

[2] S. Machida, et al., Proc. of EPAC2000, Vienna, (2000) 557-559. 